Received: 30 April 2018

Accepted: 31 August 2018

Published online: 12 September 2018

\section{Origin of relationship between ferromagnetic response and damage in stretched systems}

\author{
S. Merabtine ${ }^{1}$, F. Zighem ${ }^{1}{ }^{1}$, A. Garcia-Sanchez ${ }^{1}$, V. Gunasekaran ${ }^{1,2}$, M. Belmeguenai ${ }^{1}$, \\ X. Zhou ${ }^{2}$, P. Lupo ${ }^{2}$, A. O. Adeyeye ${ }^{2}$ \& D. Faurie ${ }^{1}$
}

This article presents a study whose purpose is to elucidate the damage effects in thin films on their magnetic response. $\mathrm{CO}_{40} \mathrm{Fe}_{40} \mathrm{~B}_{20}$ and $\mathrm{Ni}_{80} \mathrm{Fe}_{20}$ films of different nanometric thicknesses were stretched by more than $10 \%$ and in situ probed by atomic force microscopy measurements to determine their irreversible mechanical behavior (multi-cracking, buckling). Once these phenomena have been well identified, magnetic behavior of these stretched systems has been studied by ferromagnetic resonance to measure resulting magnetic anisotropy and damping evolutions. All of these experimental studies show that the magnetic properties are mainly affected by the stresses generated during the damage but not by the local discontinuities induced by the numerous cracks and buckles. This is in particular confirmed by the almost zero sensitivity to the damage of the magnetic properties of $\mathrm{Ni}_{80} \mathrm{Fe}_{20}$ alloy which is known for its vanishing magnetostriction.

Nanoscale systems fabricated on flexible or stretchable substrates (elastomers or polymers such as Kapton ${ }^{\circledR}$, PET, PDMS, PVDF, etc.) are being studied more and more because of their ability to adapt to non-planar surfaces, particularly in confined environments ${ }^{1-3}$. In addition, these systems have the advantage of being lighter and less expensive than their counterparts deposited on more conventional rigid substrates (silicon, ...). In recent years, many magneto-electronic devices have been made on different polymer substrates ${ }^{4-7}$. The ability of these magnetic thin films on polymer substrates to be folded or stretched is essential ${ }^{8-12}$, but their use is still delicate, which can be a brake on the industrialization of these systems ${ }^{13}$.

The main issues are to understand how the applied strains to the flexible magnetic systems impact their magnetic properties ${ }^{14-16}$. Obviously, when a thin film is deposited on a flexible substrate, it is usually submitted to high mechanical stresses due to the stretching or the curvature of the whole system. Moreover, these stresses are all the more important given that the Young's modulus contrast is high between the film and the substrate. This contrast is characterized by the Dundurs parameter $\alpha_{D}{ }^{17,18}$ :

$$
\alpha_{D}=\frac{\overline{Y_{f}}-\bar{Y}_{s}}{\bar{Y}_{f}+\bar{Y}_{s}}
$$

were $\bar{Y}=Y /\left(1-\nu^{2}\right)$ is the plane strain elastic modulus ${ }^{19}, \nu$ is the Poisson ratio, subscript $f$ and $s$ refer to the film and the substrate respectively. The value of $\alpha_{D}$ varies from -1 for a rigid substrate to +1 for a compliant substrate. In the last case, a small effort applied to the system lead to high stresses in the thin film.

These stresses may have an important effect on the static and dynamic magnetic properties of thin films on polymer substrates. In particular, it is important that the large deformations to which they are subject are not harmful to their functional properties. In fact, beyond the classical magnetoelastic effects observable at small strains, the phenomenon of cracking ${ }^{20,21}$ and associated localized buckling observed for inorganic thin films on organic substrates tensily stressed ${ }^{22-25}$ lead to heterogenous strains that may have effects on magnetic properties. However, these are rarely discussed and have never been studied in depth.

In this work, we focused on experimental identification of cracking mechanisms for two magnetic alloys $\left(\mathrm{Co}_{40} \mathrm{Fe}_{40} \mathrm{~B}_{20}, \mathrm{Ni}_{80} \mathrm{Fe}_{20}\right)$ deposited on Kapton ${ }^{\circledR}$ substrate $(125 \mathrm{~mm})$. The phenomena of multi-cracking but also

${ }^{1}$ LSPM-CNRS, Université Paris XIII-Sorbonne Paris Cité, 93430, Villetaneuse, France. ${ }^{2}$ Information Storage Materials Laboratory, Department of Electrical and Computer Engineering, National University of Singapore, Singapore, 117576, Singapore. Correspondence and requests for materials should be addressed to F.Z. (email: zighem@univparis13.fr) orA.O.A. (email: eleaao@nus.edu.sg) or D.F. (email: faurie@univ-paris13.fr) 


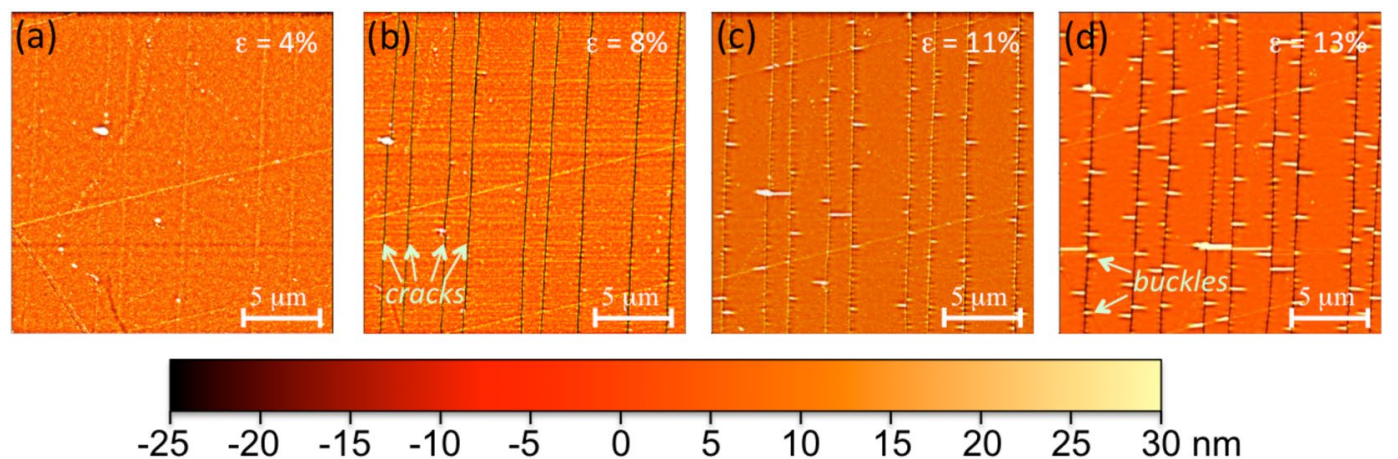

Figure 1. AFM images highlighting the multi-cracking and subsequent buckling of a CoFeB $20 \mathrm{~nm}$-film obtained at different strain states.

buckling of thin films have been studied. Thin films surface was probed by atomic force microscopy (AFM) during or before tensile tests to clearly identify these mechanisms. Beyond the mechanical properties, we then examined the magnetic properties of these systems. At first, we estimated magnetoelastic properties of the two materials by piezoelectric actuation and in situ ferromagnetic resonance (FMR). These data collected at small strains are important for interpreting results for higherstrains. Subsequently, we have identified the ex situ effects of irreversible phenomena, particularly "cracks" and "cracks-buckling" regimes, on magnetic properties of thin films (anisotropy and damping).

\section{Experimental Section}

Materials deposition. $\mathrm{Co}_{40} \mathrm{Fe}_{40} \mathrm{~B}_{20}$ (CoFeB thereafter) and $\mathrm{Ni}_{80} \mathrm{Fe}_{20}$ thin films were deposited on $125 \mathrm{~m}$ thick Kapton ${ }^{\circledR}$ substrates. In order to avoid any contamination, cleaning of these substrates was carried out in a clean room. They were immersed in isopropanol in a beaker and placed in an ultrasonic bath for 10 minutes. Following this, they were cleaned using distilled water and a nitrogen gun, before being stored in a clean plastic case. Samples were made by varying the thicknesses of the thin films from 10 to $100 \mathrm{~nm}$. On the other hand, the substrates were once again cleaned with a plasma etching $\left(\mathrm{Ar}+\mathrm{O}_{2}\right)$ at $3 \mathrm{mTorr}$ for 120 seconds just before the deposition. The deposits were made under vacuum (residual pressure of $2.10^{-8}$ Torr) at an argon pressure of 1 mTorr. This is to eliminate any organic residues and improve the adhesion of the films to the substrate. CoFeB material is known to be amorphous at room temperature and is very used in spintronics applications ${ }^{26}$, while $\mathrm{Ni}_{80} \mathrm{Fe}_{20}$ is a reference crystalline material for magnetism community because its magnetoelastic constants are close to zero ${ }^{27}$.

In situ mechanical testing. The tensile tests have been performed by using a technique combining a micro-tensile tester and AFM observations ${ }^{28,29}$. The tensile loads were applied to specimens by means of a $300 \mathrm{~N}$ Deben $^{\mathrm{TM}}$ tensile module. This tensile tester is equipped with a $300 \mathrm{~N}$ load cell enabling the force measurement with a precision of $0.1 \mathrm{~N}$. The strain rate has been kept constant, equal to $3.10^{-4} \mathrm{~s}^{-1}$ during loading ramps (up to $20 \%$, the initial length is $28 \mathrm{~mm}$ and the displacement rate is $0.5 \mathrm{~mm} / \mathrm{min}$ ). Samples are mounted horizontally, clamped to a pair of jaws and supported on stainless steel sliding bearings. A dual threaded lead screw drives the jaws symmetrically in opposite directions, keeping the sample centered in the field of view. The module is ideally suited for use with AFM. The AFM observations have been made during test breaks by using a standard Veeco D3100 microscope (in contact mode).

Ferromagnetic resonance (FMR). The FMR setup allows the determination of the resonance field $H_{\text {res }}$ of the uniform precession mode by sweeping the applied magnetic field in presence of a fixed pumping radio frequency field $\vec{h}_{r f}$ (i.e. microwave driving frequency $\left.f\right)^{30}$. In order to enhance the signal to noise ratio, a weak modulation of the static applied magnetic field (here $5 \mathrm{Oe}$ at $175 \mathrm{~Hz}$ ) is performed. Thus, in absence of applied strains, the resonance field of the uniform precession mode is expected to only depends on the gyromagnetic factor $\gamma$, the saturation magnetization $M_{s}$ and the presence of magnetic anisotropies. The presence of in-plane anisotropies can be easily detected by measuring the in-plane angular dependence of the uniform precession mode. Finally, the magnetoelastic properties of the films have been studied using the FMR setup with in situ micro-mechanical testing (at small strains, e.g. about $0.1 \%)^{31}$. For this purpose, the film/substrate systems have been glued on top of piezoelectric actuators. The principe of this in situ measurements is to deform the films through the voltage applied to the actuator, the strains being perfectly transmitted from the actuator to the films. The resonance fields are measured by FMR while the strains are measured by digital image correlation (DIC) from optical tracking of the mottled back surface ${ }^{32,33}$.

\section{Experimental Results and Discussions}

Mechanical properties. In this section, we focuse on experimentally identifying cracking mechanisms for the two studied alloys $\left(\mathrm{CoFeB}\right.$ and $\mathrm{Ni}_{80} \mathrm{Fe}_{20}$ ) deposited as thin films on Kapton ${ }^{\circledR}$ substrate $(125 \mathrm{~mm})$. Figure 1 shows images of the surface of a $20 \mathrm{~nm}$ thick $\mathrm{CoFeB}$ thin film for four applied strain states in order to understand the cracking mechanism. The goal here is to identify the cracks morphology that appear within the films. We tried to record our images on the same region of the film although drifts of a few micrometers are inevitable in 


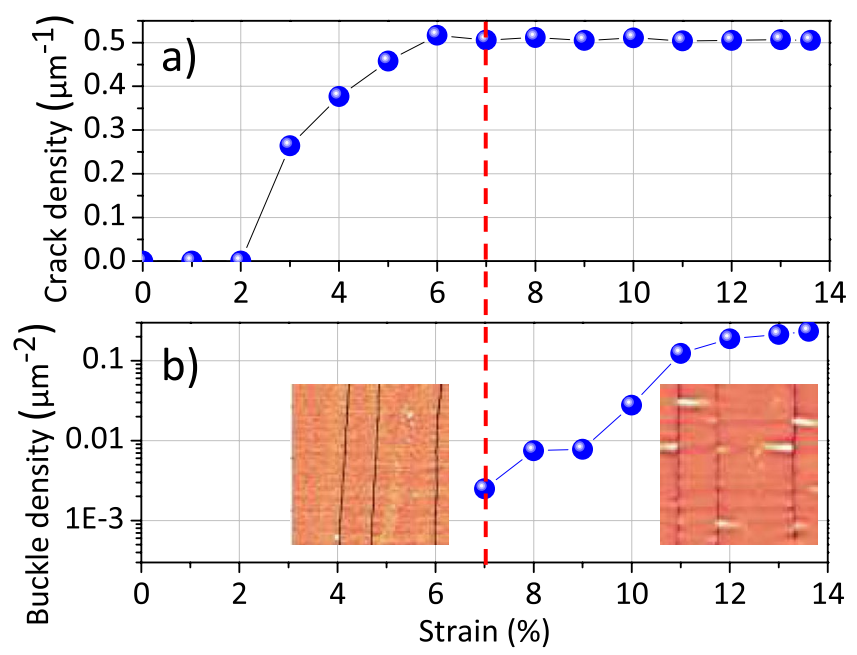

Figure 2. Variations of the crack density (a) and of the buckle density (b) as a function of the macroscopic deformation applied to the substrate for a $20 \mathrm{~nm} \mathrm{CoFeB} \mathrm{film;} 6 \times 6 \mathrm{~m}^{2}$ AFM images have been inserted for illustration.

that feedback loop is not available on the microscope. It is observed that straight cracks appear from a strain below $5 \%$ and that they multiply during the deformation. Finally, after $8 \%$ of strain, blisters are observed which are initiated at the edge of previously created cracks with a stop of the multiplication of the latter, which will be quantified thereafter. Kapton ${ }^{\circledR}$ material is known to deform plastically from $4-5 \%$ of macoscopic strain. Actually, the plastic deformation of the substrate should be localized between the film fragments induced by $\mathrm{cracks}^{34}$ that may enhance the buckling phenomenon.

Figure 2a shows the evolution of the linear density of cracks as a function of the macroscopic strain applied to the substrate for the $20 \mathrm{~nm} \mathrm{CoFeB}$ films. It increases from a strain of $2 \%$ until saturation after $6 \%$. In addition, it can be seen in 2-b that the surface density of blisters becomes non-zero when the crack density begins to saturate, then increases strongly up to $14 \%$. Thus, these in situ experiments show that we are in the presence of two damage regimes: (i) multiplication of cracks and (ii) multiplication of blisters. In order to highlight these two regimes on the same graph, we plotted the surface density of blister $\rho$ multiplied by the square of the thickness of the film $t_{f}^{2}$ as a function of the linear density of cracks. The choice to multiply by $t_{f}^{2}$ is related to the strong effect of thickness on $\rho$ and thus makes it possible to compare several thicknesses on the same graph.

The data corresponding to the previous sample loaded in situ is in Fig. 3a) in open blue symbol. It is clear that the parameter $\rho t_{f}^{2}$ only increases when the density of cracks reaches saturation of $0.5 \mathrm{~m}^{-1}$. For reasons of space, the magnetic measurements whose results are presented in a next paragraph can only be ex situ performed. We have therefore undertaken ex situ AFM measurements for different maximum strains achieved which we have compared with previously realized in situ measurements, for the thickness of $20 \mathrm{~nm}$. Very consistent results are observed (comparison between full and open blue symbols) since the phenomena of cracking and delamination are irreversible.

Further ex situ AFM experiments after tensile tests were performed for different thicknesses $(10 \mathrm{~nm}, 50 \mathrm{~nm}$ and $100 \mathrm{~nm}$ ) shown respectively in red, green and black symbols in Fig. 3a). It is observed that the maximum density of cracks decreases with the thickness (from $0.75 \mathrm{~m}^{-1}$ for $10 \mathrm{~nm}$ to $0.20 \mathrm{~m}^{-1}$ for $100 \mathrm{~nm}$ ), as already described by shear lag models ${ }^{35}$. In addition, the surface density of blisters also decreases with thickness, which results in a slight variation of the term $\rho t_{f}^{2}$. In contrast, this behavior has been poorly described in the litterature. Being given that buckling intiate at the edge of cracks, it is obvious that a higher saturating crack density (for thinner films) must lead to higher blister density.

This therefore reflects an increase in the sources of strain heterogeneities when the thickness decreases (greater density of damages) which will have to result in an evolution of the magnetic properties if the magnetoelastic coupling is significant, as will be shown later. This study of the effect of thickness was also carried out for $\mathrm{Ni}_{80} \mathrm{Fe}_{20}$ alloy in Fig. 3b). The same bimodal behavior and the same effect of thickness are observed with, however, slightly lower maximum cracks density values for all the thicknesses. This alloy is also subject to severe damage during uniaxial tests with comparables phenomena.

Magnetic and magnetoelastic characterization. The "standard" characterization of thin films by magnetic resonance often begins with the measurement of the angular dependence of the resonance field in order to verify the presence of planar anisotropies and their orders ${ }^{36}$. In this regard, Fig. 4 shows the angular dependencies of the resonance field $H_{\text {res }}$ of the $20 \mathrm{~nm}$ thick $\mathrm{CoFeB}$ and $\mathrm{Ni}_{80} \mathrm{Fe}_{20}$ films measured at $8 \mathrm{GHz}$. The difference in the average resonance field value for the two materials is simply due to the saturation magnetization difference. In addition, there is the presence of a uniaxial anisotropy (order 2) for the two materials. This residual uniaxial anistotropy $H_{u}$ can be calculated with $2 H_{u}=H_{\text {res }}^{\text {max }}-H_{\text {res }}^{\text {min }}$. Gueye et al. ${ }^{37}$ have shown that in the case of compliant substrates this uniaxial anisotropy is due to the slight curvature of the film/substrate systems subjected to residual 


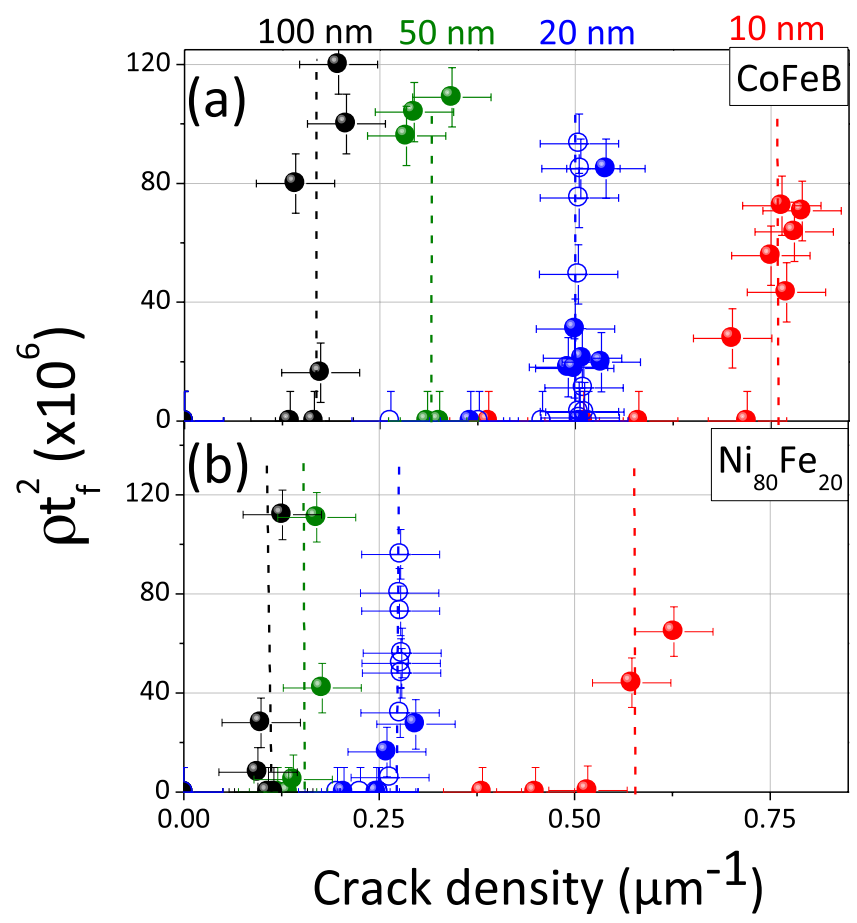

Figure 3. Variations of the surface density of blister $\rho$ multiplied by squared thickness of the film as a function of the linear density of cracks highlighting the two different regimes (multi-cracking and buckles). Graph a) corresponds to data for $\mathrm{CoFeB}$ films and graph b) to date for $\mathrm{Ni}_{80} \mathrm{Fe}_{20}$ films.

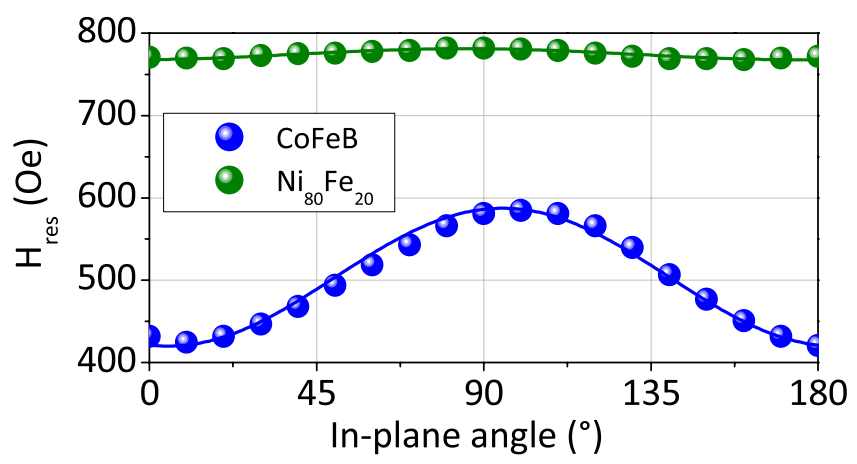

Figure 4. Angular dependence of the resonance field for $\mathrm{CoFeB}$ (blue) and $\mathrm{Ni}_{80} \mathrm{Fe}_{20}$ (green) $20 \mathrm{~nm}$-films. Solid lines correspond to fits obtained using Eq. 1 with the parameters indicated in the text.

stresses. Thus, this anisotropy is weaker as the magnetostriction coefficient is low. Indeed $\mathrm{Ni}_{80} \mathrm{Fe}_{20}$ is known to have a very weak magnetostriction coefficient that is consistent with the very weak measured value of $H_{u}(7 \mathrm{Oe})$ as compared to one measured for $\mathrm{CoFeB}(85 \mathrm{Oe})$. In theses conditions and by asuming that the magnetization is always aligned along the applied magnetic field during the angular rotation, the resonance field angular dependencies can be adjusted using:

$$
H_{r e s}=\sqrt{\left(2 \pi M_{s}+H_{u} \sin \varphi_{H}^{2}\right)^{2}+\left(\frac{2 \pi}{\gamma}\right)^{2}}-2 \pi M_{s}-H_{u}\left(\frac{1}{2}+\frac{3}{2} \cos 2 \varphi_{H}\right)
$$

Where $\varphi_{H}$ corresponds to the in-plane angle (referring to the film edges), $M_{s}$ is the saturation magnetization, $f$ is the driving frequency and $\gamma$ is the gryomagnetic ratio. Solid lines in Fig. 4 refer to fits using $M_{s}=1040 \mathrm{emu} \mathrm{cm}^{-3}$ (resp. 755); $\gamma=1.948 \times 10^{7} \mathrm{~Hz}^{-O \mathrm{Oe}^{-1}}$ (resp. $1.835 \times 10^{7}$ ) and $H_{u}=85 \mathrm{Oe}$ (resp. $7 \mathrm{Oe}$ ) for the CoFeB $20 \mathrm{~nm}$-film (resp. $\mathrm{Ni}_{80} \mathrm{Fe}_{20}$ ). The fitting results are in good agreement with the experimental ones. We have extented this "standard" characterization to all films (all thicknesses). Those measurements show that $M_{s}, H_{u}$ and $\gamma$ are almost constant as function of the film thickness for both the alloys (see Table 1).

In addition, the determination of the magnetostriction coefficient is also an important parameter if one wants to analyze, even qualitatively, the results obtained in the context of large strains (next section). This determination was carried out following the methodology developed in $^{32}$ by making the hypothesis of elastic (Young's modulus 


\begin{tabular}{|c|c|c|c|c|}
\hline Thickness (nm) & $M_{s}\left(\mathrm{emu} \mathrm{cm}^{-3}\right)$ & $H_{u}(\mathrm{Oe})$ & $\gamma\left(\times 10^{7}\right) \mathrm{Hz} \cdot \mathrm{Oe}^{-1}$ & $\lambda\left(\times 10^{-6}\right)$ \\
\hline \multicolumn{5}{|l|}{$\mathrm{Ni}_{80} \mathrm{Fe}_{20}$} \\
\hline 10 & 750 & 7 & 1.835 & $\mathrm{~nm}$. \\
\hline 20 & 755 & 7 & 1.835 & 0.72 \\
\hline 50 & 770 & 6 & 1.835 & $\mathrm{~nm}$. \\
\hline 100 & 790 & 6 & 1.835 & 0.75 \\
\hline \multicolumn{5}{|l|}{$\mathrm{Co}_{40} \mathrm{Fe}_{40} \mathrm{~B}_{20}$} \\
\hline 10 & 1030 & 74 & 1.948 & 22 \\
\hline 20 & 1040 & 85 & 1.948 & 23 \\
\hline 50 & 1080 & 84 & 1.948 & $\mathrm{~nm}$. \\
\hline 100 & 1095 & 95 & 1.948 & 24 \\
\hline
\end{tabular}

Table 1. Saturation magnetization $\left(M_{s}\right)$, anisotropy field $\left(H_{u}\right)$, gyromagnetic factor $(\gamma)$ and magnetostriction coefficient $(\lambda)$ of $\mathrm{CoFeB}$ and $\mathrm{Ni}_{80} \mathrm{Fe}_{20}$ films. $\mathrm{nm}$. means not measured. The absolute uncertainty for $\lambda$ is about $0.05 \times 10^{-6}$.

a)
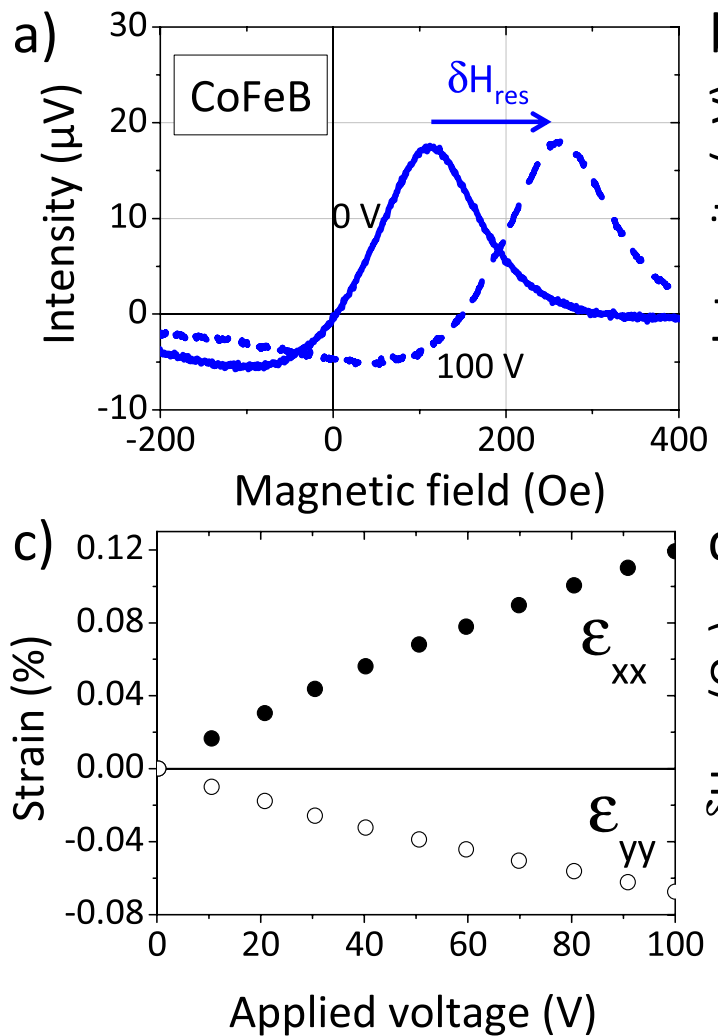

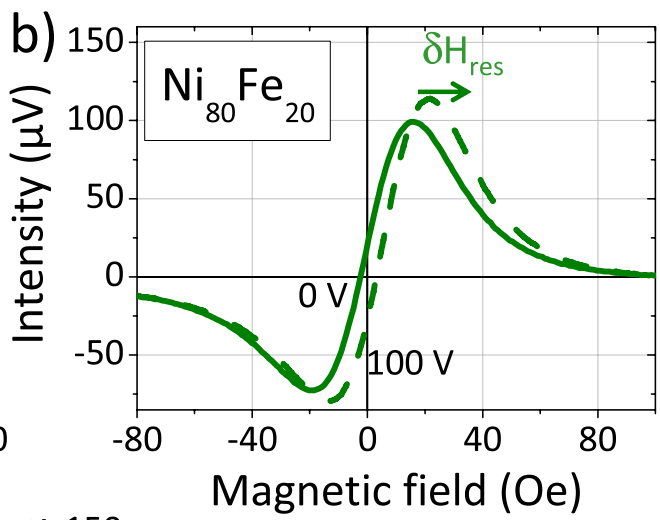

d)

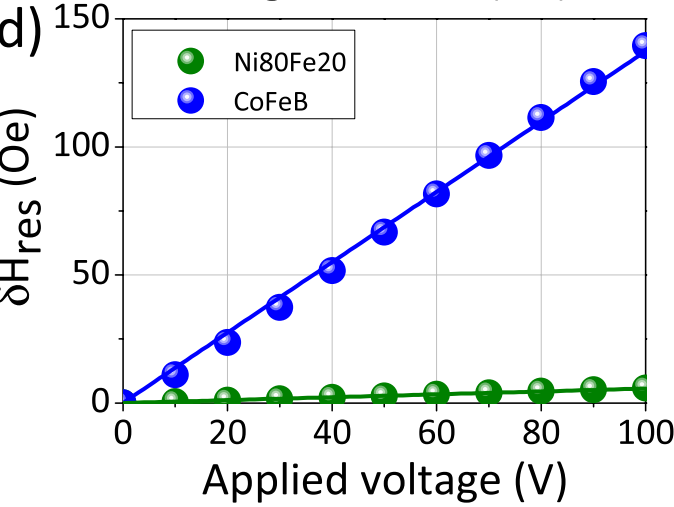

Figure 5. (a,b) $\mathrm{Ni}_{80} \mathrm{Fe}_{20}$ and $\mathrm{CoFeB} 20 \mathrm{~nm}$-films resonance spectra for two different voltages applied to the piezoelctronic actuators $(0 \mathrm{~V}$ and $100 \mathrm{~V})$. (c) Variation of the in-plane strains $\left(\varepsilon_{x x}\right.$ and $\left.\varepsilon_{y y}\right)$ measured at the top surface of the films as a function of the applied voltage d) Resonance field shift $\left(\delta H_{\text {res }}=H_{\text {res }}(100 \mathrm{~V})-H_{\text {res }}(0)\right)$ variations as function of the applied voltage. Solid lines refer to the adjusting models for the two samples, using Eq. 2 with the parameters indicated in the text.

$Y$ and Poisson's ratio $\nu$ ) and magnetoelastic (magnetostriction $\lambda$ ) isotropy. For this purpose, we cemented the film/substrate system on a piezoelectric actuator. The principle is to record resonance spectra at different strain states by applying a voltage to the piezoelectric actuator. The transmission of planar strains from the actuator to the thin film is measured by digital image correlation (DIC) and is usually $100 \%$ in the case of compliant substrates $^{31,38}$. Figure $5 \mathrm{c}$ ) gives the correspondance between the applied voltage and the transmitted in-plane strains $\left(\varepsilon_{x x}\right.$ and $\left.\varepsilon_{y y}\right)$ extracted from DIC measurements. We can see here that $\varepsilon_{x x}$ is positive while $\varepsilon_{y y}$ is negative, the accuracy being less than $10^{-5}$ in our conditions, as already reported in ${ }^{38}$. Figure $5 \mathrm{a}$,b) show typical resonance spectra of the $20 \mathrm{~nm} \mathrm{CoFeB}$ and $\mathrm{Ni}_{80} \mathrm{Fe}_{20}$ films at $0 \mathrm{~V}$ and $100 \mathrm{~V}$ obtained under similar conditions (frequency of $8 \mathrm{GHz}$ and magnetic field applied perpendicular to the actuator, i. e. perpendicular the principal elongation direction). A positive energy shift $\left(\delta H_{\text {res }}\right)$ defined as $\delta H_{\text {res }}=H_{\text {res }}(100 \mathrm{~V})-H_{\text {res }}(0)$ of the spectra is clearly observed when a voltage is applied. This shift is less intense in $\mathrm{Ni}_{80} \mathrm{Fe}_{20}$ (around $5 \mathrm{Oe}$ ) as compared to CoFeB film (around $140 \mathrm{Oe}$ ) and 


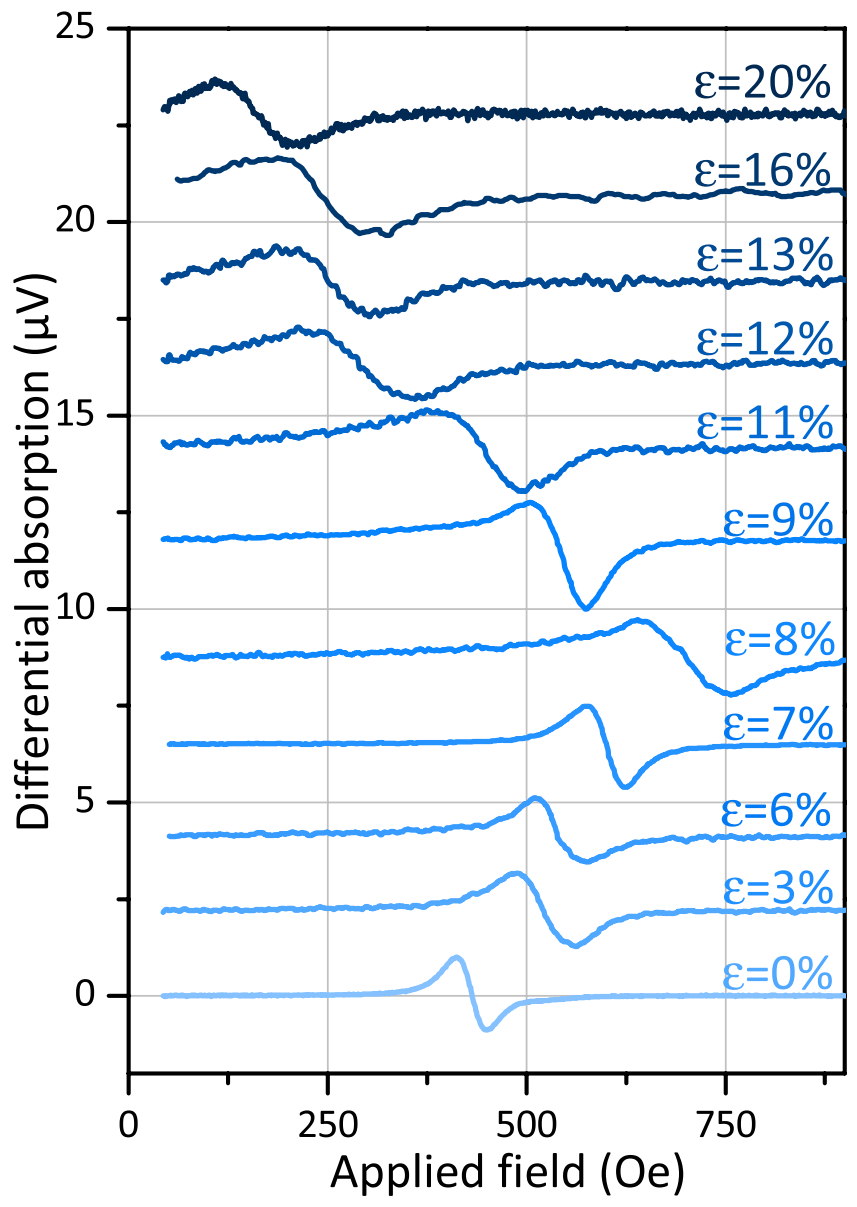

Figure 6. Typical FMR spectra of a $20 \mathrm{~nm}$ CoFeB film for different previously applied strains. Spectra have been normalized for clarity. The sketch shows the direction of the applied magnetic field (perpendicular to the preapplied traction).

is due to a lower (positive) value of $\lambda$. The quantitative determination of $\lambda$ is possible by adjusting $\delta H_{\text {res }}$ evolution as a function of the applied voltage. This evolution is shown in Fig. 5d) for both films. Since the magnetic field is applied parallel to the elongation direction of the actuator, Eq. 1 is only slightly modified ${ }^{31}$ :

$$
\begin{aligned}
H_{r e s}= & \sqrt{\left(2 \pi M_{s}+H_{u} \sin \varphi_{H}^{2}+\frac{3 \lambda}{2 M_{s}} \sigma_{x x}\right)^{2}+\left(\frac{2 \pi}{\gamma}\right)^{2}} \\
& -2 \pi M_{s}-H_{u}\left(\frac{1}{2}+\frac{3}{2} \cos 2 \varphi_{H}\right)+\frac{3 \lambda}{2 M_{s}}\left(\sigma_{x x}-2 \sigma_{y y}\right)
\end{aligned}
$$

$\sigma_{x x}$ and $\sigma_{y y}$ are the in-plane principal stress tensor components in the films which are simply given by the Hooke's law. For that purpose, $Y$ and $\nu$ values used for $\mathrm{CoFeB}$ and $\mathrm{Ni}_{80} \mathrm{Fe}_{20}$ films have been extracted from refs ${ }^{39,40}$. By adjusting Eq. 2 with the experimental data and knowing physical parameters (see Table 1), we can estimate $\lambda$ with accuracy. The deduced magnetostriction values are $23 \mathrm{ppm}$ for $\mathrm{CoFeB}$ and $0.7 \mathrm{ppm}$ for $\mathrm{Ni}_{80} \mathrm{Fe}_{20}$. Note that these values have not been systematically measured for all the other thicknesses but it is known that the thicknesses involved in this work have a negligible influence on the magnetoelastic properties ${ }^{41}$ (see Table 1).

Large strain effects on magnetic anisotropy. We will now analyze the influence of stretching on the magnetic properties of the studied films. As previously mentioned, it is important to note that FMR measurements have been performed ex situ. This is due to geometric constraints, it is indeed not easy to incorporate the tensile machine within the gap of our electromagnet. However, since we are investigating irreversible phenomena, we have analyzed previously deformed ex situ films at several levels of deformation. We have thus produced several tensile specimens of the same size and have minimized the time between applied deformation and resonance measurements (a few minutes) to minimize post-test crack closure phenomena. We have performed angular dependence measurements in order to follow the evolution of planar magnetic anisotropies. The driving frequency is fixed at $8 \mathrm{GHz}$ throughout this section. We first present results from CoFeB films. Typical FMR spectra of $20 \mathrm{~nm} \mathrm{CoFeB}$ films are presented in Fig. 6 . These spectra are obtained by applying a magnetic field transversely to the traction previously applied. It is remarkable to note that even after the application of large strains (up to 


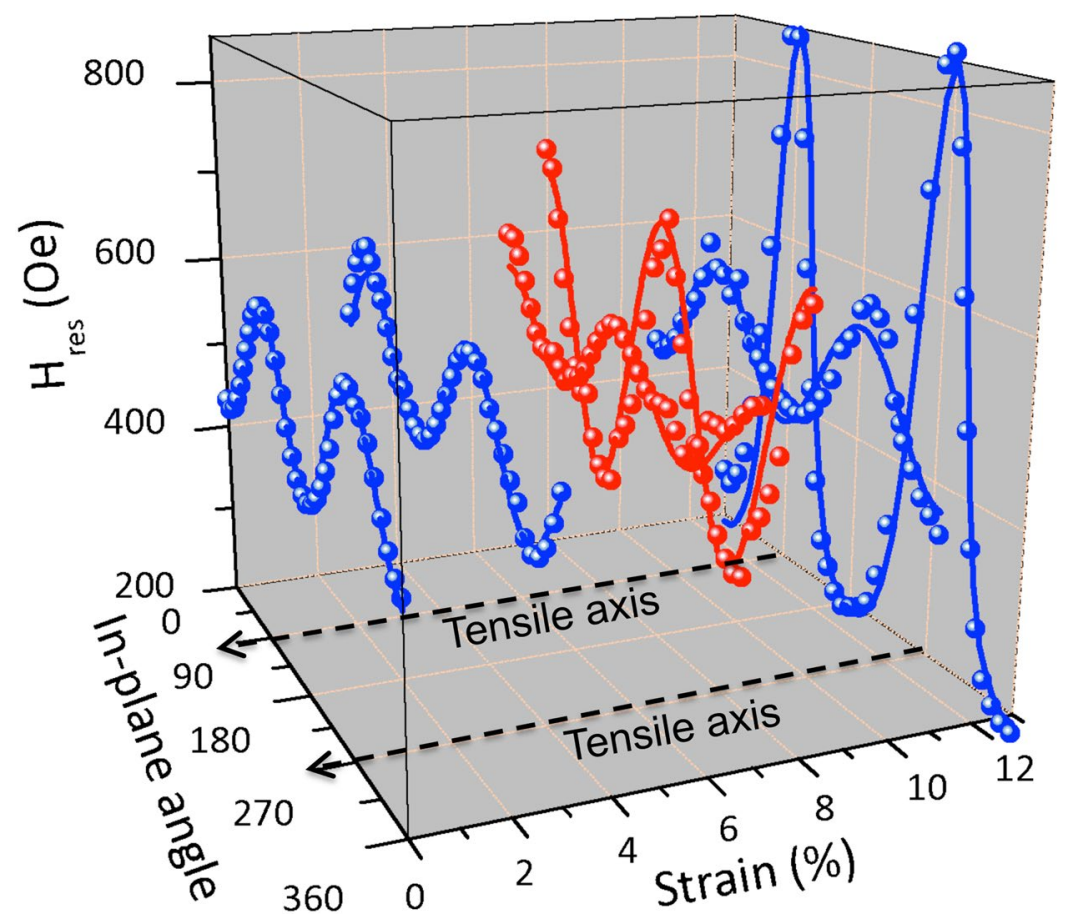

Figure 7. Resonance field $\left(H_{\text {res }}\right)$ angular dependencies for $20 \mathrm{~nm}$ CoFeB films at different previously applied strain. The blue/red color code is used to display the tilting of the direction of the easy axis. Continuous lines refer to the adjusting models for the two samples, using Eq. 1 with $M_{s}$ and $\gamma$ values previously determined, the only adjustable parameter is $H_{u}$.

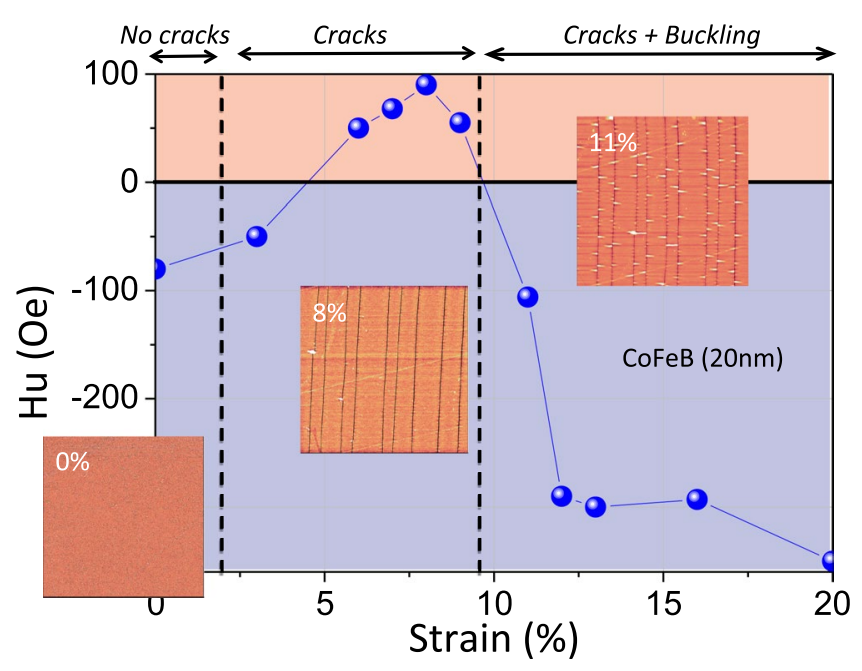

Figure 8. Variation of $H_{u}$ as a function of the strain for $20 \mathrm{~nm} \mathrm{Co}_{40} \mathrm{Fe}_{40} \mathrm{~B}_{20}$ films. $H_{u}$ is counted positively when it is aligned along the traction and negatively when it is perpendicular to it. The AFM images $\left(20 \times 20 \mathrm{~mm}^{2}\right)$ presented are characteristic of the three regimes.

$20 \%)$, the signal is preserved. Moreover, we realize that the resonance field evolves strongly from one spectrum to another. This is due to the variation of the planar anisotropy of the films. Thus, Fig. 7 shows the angular dependencies of the resonance field for different strains applied to $20 \mathrm{~nm}$ CoFeB films. In these angular dependencies, the tensile stress is applied at $90^{\circ}$. In absence of strain (0\%), the easy axis is at $0^{\circ}$. Beyond $6 \%$, the easy axis is found along the applied stress. We used a red/blue color code to illustrate this directional tilting of the easy axis. Beyond $11 \%$, the easy axis returns to $0^{\circ}$. Moreover, in addition to the tilting of this axis of easy magnetization from $0^{\circ}$ to $90^{\circ}$ and then again to $0^{\circ}$, it is noted that the value of the anisotropy field also varies.

Figure 8 represents the variation of the deduced uniaxial anisotropy field $H_{u}$ as function of the applied strain. We used the following convention for the sign of this field: it is positive when the easy axis is along the traction and negative when this axis is at $90^{\circ}$ of the traction. This curve illustrates the results obtained from the angular 
dependences of the resonance field. It is clear that it is initially negative, then positive between $5 \%$ and $10 \%$ and then again negative beyond $11 \%$.

In order to explain this complex behavior with strain, a first assumption could be the effect of a shape anisotropy linked to the parallel nature of cracks between them. Indeed, if we see this set of fragments as an array of nanowires, this could explain the shift towards negative values ${ }^{42}$. However, the precedent subsection shows that the appearance of cracks occurs at less than $2 \%$ of deformation and the saturation of the width of the fragments is obtained at $6 \%$ of strain, values for which $H_{u}$ is still positive, which tends to invalidate this hypothesis. Moreover, an elementary magnetostatic calculation ${ }^{43}$ shows that such a shape anisotropy field is significant only for lateral dimensions smaller than those of our fragments (rather a hundred nanometers whereas our fragments have a width of about $2 \mathrm{~mm}$ ).

Furthermore, solid lines in Fig. 7 are fits to the experimental data. These fits have been plotted using Eq. 1 and by using $M_{s}$ and $\gamma$ values of the unstrained state (see Table 1), the only adjutable parameter is $H_{u}$. The good agreement between fits and experimental data shows that $M_{s}$ and $\gamma$ values are not affected by large strains and subsequent fragmentation of the films. In these conditions, we have no reason to think either that the magnetostriction coefficient $\lambda$ changes strongly or that it changes sign.

We have to find a better correlation with the results obtained in the precedent subsection. We have highlighted the presence of 3 distinct regimes for these CoFeB films: (i) elastic domain, (ii) crack multiplication, (iii) decohesion with fixed crack density. Figure 8 shows which ranges of strains correspond to these regimes. We also show a typical AFM image $\left(20 \times 20 \mathrm{~mm}^{2}\right)$ of each of these regions. We will discuss the $H_{u}$ variations, based on the planar stress state induced by each tensile test. Indeed, we know that the magnetoelastic contribution to the anisotropy field can be simply written as a function of planar stresses ${ }^{32}$ :

$$
H_{u}=\frac{3 \lambda}{M_{s}}\left(\sigma_{x x}-\sigma_{y y}\right)
$$

We are in presence of ex situ measurements. Thus, any magnetoelastic contribution to the anisotropy field can only be due to residual stresses generated during the tensile test and during the elastic discharge of the substrate. First, it is clear that the multiplication of cracks is correlated with the first $H_{u}$ change of sign. Indeed, for fragile films, it is known in this regime that a longitudinal stress plateau is observed while a transverse compressive stress develops and increases as long as no decohesion of the film occurs. This phenomenon is illustrated in Fig. 7 of ref. ${ }^{34}$ for Ta- $\alpha$ films for which the stresses are in situ measured by $\mathrm{x}$-ray diffraction. We believe that this difference between $\sigma_{x x}$ and $\sigma_{y y}$ is sufficiently important for reminiscence in the residual state after discharge. This explains the positive sign of $H_{u}$ before onset of decohesion. After appearance of first decohesions, the transverse stress of compression decreases strongly in magnitude while the longitudinal stress of tension decreases and can tend towards zero for strains of $20 \%$ as also shows in Fig. 7 of ref. ${ }^{34}$. Then, a discharge of the substrate from such a state of stress in the film can quite reverse the sign of transverse and longitudinal stresses. Since we have not identified any other source of modification for a magnetoelastic contribution, we believe that such a reversal of the stresses has occurred.

In order to confirm our observations on these $20 \mathrm{~nm}$ CoFeB films, we performed tensile tests followed by magnetic resonance measurements on the other CoFeB films (10, 50 and $100 \mathrm{~nm})$. The results from angular dependence measurements are presented in Fig. 9. In this figure, the dashed black vertical lines delimit the three regimes that we have identified through in situ AFM observations. It is immediately interesting to note that the regime "cracks + buckling" starts approximately for the same strain ( 9\%) while "crack initiation" regime is dependent on the thickness of the film. This initiation starts at $2 \%$ for films of 10 and $20 \mathrm{~nm}$, at $\sim 4 \%$ for $50 \mathrm{~nm}$ films and at $\sim 6 \%$ for $100 \mathrm{~nm}$ films. We note that the first sign inversion of $H_{u}$ is dependent on the films thickness. The strain for which this inversion appear increase with the films thickness: $\sim 3-4 \%$ for $10 \mathrm{~nm}$; at $\sim 5-6 \%$ for $20 \mathrm{~nm}$; at $\sim 6-7 \%$ for $50 \mathrm{~nm}$ and at $\sim 7-8 \%$ for $100 \mathrm{~nm}$. This inversion is strongly correlated with the appearance of the first cracks which is also dependent on the thickness. There is an inertia between the appearance of the cracks and the first sign inversion for each thickness This is not surprising since it is probably necessary to achieve a sufficient density of cracks to generate the state of residual stresses necessary to change the $H_{u}$ sign.

On the other hand, it is interesting to note that the second sign inversion takes place roughly for the same strains whatever the thickness, which again is well correlated with the delimitation between the "crack" and "cracks + buckling" regimes. Finally, concerning the $H_{u}$ value, we note that the minimum and maximum values of $H_{u}$ are approximately same (regardless of the strains for which sign inversions occur) whatever the thickness and therefore the width of the fragments. Moreover these values of the order of 300-400 Oe are much higher than those expected for the possible shape anisotropy which definitively exclude this hypothesis. We conducted similar experiments with $\mathrm{Ni}_{80} \mathrm{Fe}_{20}$ films. Their $H_{u}$ variations are presented in Fig. 9 (open symbols). One can note here that these $H_{u}$ values come from adjustments of the angular dependencies of $H_{\text {res }}$. As for CoFeB, $M_{s}$ and $\gamma$ values do not seem to be affected by these large deformations. We observe that $H_{u}$ is almost constant for $\mathrm{Ni}_{80} \mathrm{Fe}_{20}$ films even if similar damages (multicracking and buckling) are also observed. Thus, we attribute this monotony of $H_{u}$ as a function of strains to the almost zero magnetostriction coefficient for this alloy, thus confirming that these results must be analyzed in the context of residual stresses and that contributions other than magnetoelastic could be neglected (such as magnetostatic contributions due to breaking translation invariance).

Large strain effects on magnetic damping. Finally, we have used the spectra recorded at $8 \mathrm{GHz}$ to analyse the evolution of the magnetic damping parameter, which is of strong importance in spintronic applications since it governs the speed at which the magnetization can be reversed or reoriented. In a fixed frequency field-swept FMR experiment, the peak to peak linewidth $\Delta H_{p p}$ is proportional to the frequency with a slope determined by ${ }^{44,45}$ : 


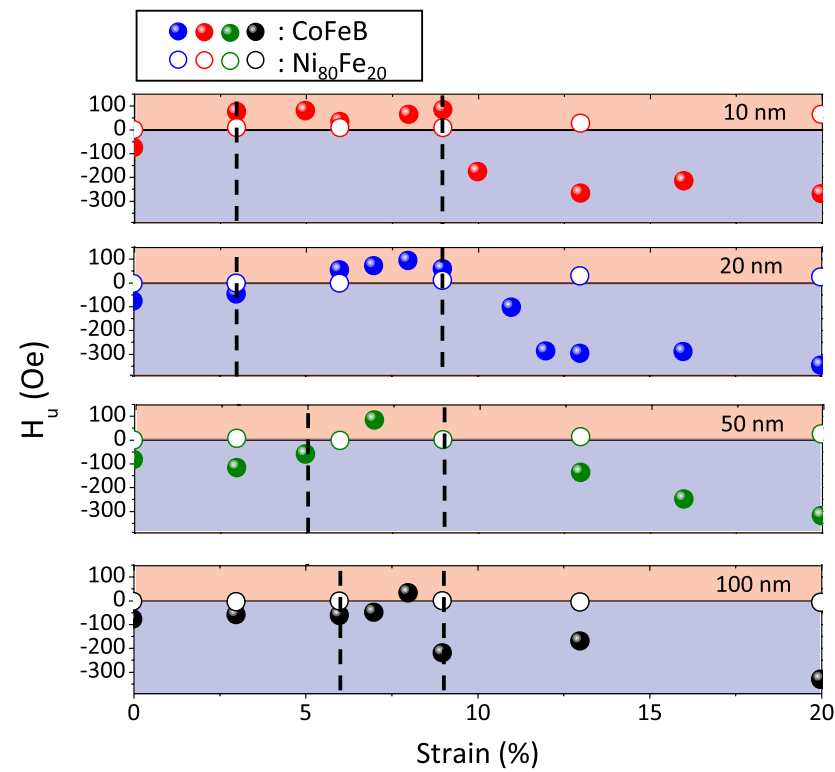

Figure 9. $H_{u}$ variations as a function of the applied strains for $\mathrm{CoFeB}$ (filled symbols) and $\mathrm{Ni}_{80} \mathrm{Fe}_{20}$ (open symbols) films of different thicknesses: $10,20,50$ and $100 \mathrm{~nm}$. This field is counted positively when it is aligned along the traction and negatively when it is perpendicular to it. The $\mathrm{Co}_{40} \mathrm{Fe}_{40} \mathrm{~B}_{20}$ films regimes ("absence of cracks", "cracks" and "cracks + buckling") are delimited by vertical black dotted lines.
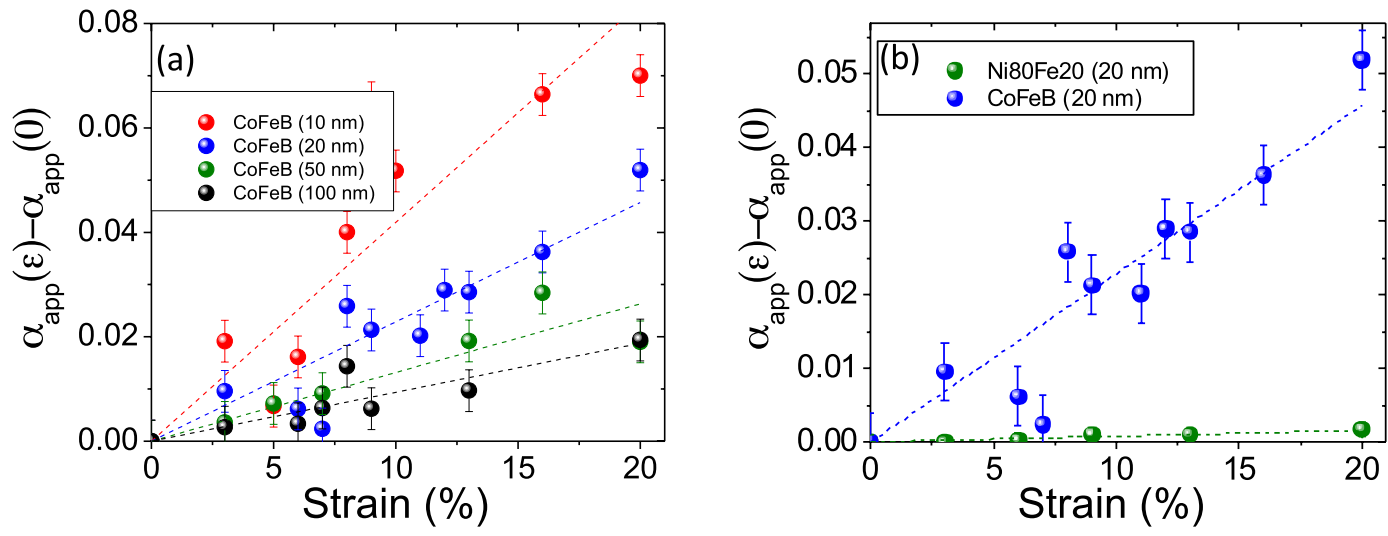

Figure 10. (a) Evolution of the apparent damping $\alpha_{a p p}$ as a function of the previously applied strains for CoFeB films. (b) Variation of $\alpha_{a p p}$ as a function of the previously applied strains for $20 \mathrm{~nm}$ films of both materials (CoFeB and $\mathrm{Ni}_{80} \mathrm{Fe}_{20}$ ).

$$
\Delta H_{p p}=\Delta H_{0}+\frac{2 \alpha}{\sqrt{3} \gamma} 2 \pi f
$$

where $\Delta H_{0}$ is referred to inhomogeneous contributions to the linewidth (that can be also frequency dependent ${ }^{46}$ ) and $\alpha$ is the magnetic damping. This equation is only valid when the magnetization is aligned along the applied magnetic field, which is almost the case for our analyzed spectra. In the following; for the sake of brevity, we define an "apparent" damping parameter $\alpha_{a p p}$ deduced from the mean values of $\Delta H_{p p}$ as function of the in-plane angle (at $8 \mathrm{GHz}$ ). As we are interested in the evolution of the apparent damping as function of the strain, we have plotted $\alpha_{a p p}(\varepsilon)-\alpha_{a p p}(0)$ (where $\alpha_{a p p}(0)$ is the value of the unstrained films) as function of the pre-applied strain in Fig. 10a) for CoFeB films. Figure 10b compares the $\alpha_{a p p}$ evolution for the two studied alloys ( $20 \mathrm{~nm}$ films). The $\alpha_{\text {app }}$ parameter is the signature of the heterogeneities present within the films. Thus it will depend on the strains heterogeneities and will be exacerbated by the average strain. In term of magnetism, it corresponds to the heterogeneity of $H_{u}$ exacerbated by its average value. Note that this coefficient has a globally increasing dependence for both materials as function of the strain. The dotted lines are there to mark this trend. For CoFeB films, we note that the greater the thickness, the less the slope of these lines is important (see Fig. 10a)). This is consistent with the previous results of this work. Indeed, the thinnest films have cracks densities and decohesions the most 
important. They are sources of strong heterogeneities. We remember that the evolution of the average value of $H_{u}$ was rather weakly dependent on the thickness, it is therefore a pure effect of heterogeneity. In fact, if these increases were due only to an increase (in absolute value) of the average anisotropy field, the final values of $\alpha_{a p p}$ would all be the same at $20 \%$ of deformation, which is not the case. In addition, in these evolutions, we note the influence of the contribution of the average value of $H_{u}$. Indeed, if we look closely at the CoFeB curve, we can see the signature of the "cracks" region that we observed for $H_{u}$ (softening of $\alpha_{a p p}$ between $2 \%$ and $7 \%$ that we see particularly well for $10 \mathrm{~nm}$ and $20 \mathrm{~nm}$ films).

For the $\mathrm{Ni}_{80} \mathrm{Fe}_{20}$ films, we notice that high strains have only small relative influence on $\alpha_{a p p}$ values. This is quite remarkable when one puts these results in relation to the multiple fissures and decohesions present within these films. Indeed, the low magnetostriction makes this material insensitive from a magnetic point of view to the strains heterogeneities. This conclusion is highlighted by Fig. 10b) where we clearly see the small effect of these large strains on $\alpha_{a p p}$ compared to CoFeB films.

\section{Conclusion}

Two materials commonly used in the field of spintronics $\left(\mathrm{Co}_{40} \mathrm{Fe}_{40} \mathrm{~B}_{20}, \mathrm{Ni}_{80} \mathrm{Fe}_{20}\right)$ have been deposited on a Kapton ${ }^{\circledR}$ substrate, with variable thicknesses (from $10 \mathrm{~nm}$ to $100 \mathrm{~nm}$ ). We have shown that these films have two distinct irreversible deformation regimes, the first being related to the multiplication of cracks and the other to the multiplication of blisters. Overall, the density of these damages is increasing when the thickness decreases. Subsequently, we identified the ex situ effects of large strains and particularly of the "cracks" and "cracks + buckling" regimes. In the case of $\mathrm{Co}_{40} \mathrm{Fe}_{40} \mathrm{~B}_{20}$ films, the magnetic anisotropy field undergoes two sign changes, the first being attributed to the residual stress field related to the crack distribution and the second to the strong transverse stress relaxation when buckling occurs. In contrast, the anisotropy remains stable for $\mathrm{Ni}_{80} \mathrm{Fe}_{20}$, which is attributed to its low magnetostriction coefficient. Moreover, one notes a strong increase of the damping as a function of the macroscopic strain applied to $\mathrm{Co}_{40} \mathrm{Fe}_{40} \mathrm{~B}_{20} / \mathrm{Kapton}{ }^{\circledR}$ systems. This was very little observed for the $\mathrm{Ni}_{80} \mathrm{Fe}_{20}$. Indeed, magnetic damping is directly related to the magnetic field heterogeneities in the material. In this case, this is the magnetoelastic field heterogenity, related to strain heterogenities due to damages, which induces the increase of damping. Thus, a material which magnetic properties are insensitive to strains by nature has the remarkable property of behaving in a substantially similar manner whether it is "intact" or exhibiting high densities of cracks and blisters. This is undoubtedly due to the fact that the inter-cracks distance and the buckles size are a little too high to induce significant anisotropies linked to geometrical features.

\section{References}

1. Kaltenbrunner, M. et al. Ultrathin and lightweight organic solar cells with high flexibility. Nature Communication 3, 770 (2012).

2. Li, L. et al. A Solution Processed Flexible Nanocomposite Electrode with Efficient Light Extraction for Organic Light Emitting Diodes. Scientific Reports 4, 4307 (2014).

3. Das, S., Gulotty, R., Sumant, A. V. \& Roelofs, A. Correction to All Two-Dimensional, Flexible, Transparent and Thinnest Thin Film Transistor. Nano Letters 14, 2861-2866 (2014).

4. Lin, G. et al. A highly flexible and compact magnetoresistive analytic device. Lab Chip 14, 4050 (2014).

5. Kim, S., Jeong, H. Y., Kim, S. K., Choi, S.-Y. \& Lee, K. J. Flexible Memristive Memory Array on Plastic Substrates. Nano Letters 11, 5438-5442 (2011).

6. Donolato, M., Tollan, C., Porro, J. M., Berger, A. \& Vavassori, P. Flexible and Stretchable Polymers with Embedded Magnetic Nanostructures. Advanced Materials 25, 623-629 (2013).

7. Makarov, D., Melzer, M., Karnaushenko, D. \& Schmidt, O. G. Shapeable magnetoelectronics. Applied Physics Review 3, 011101 (2016).

8. Bedoya-Pinto, A., Donolato, M., Gobbi, M., Hueso, L. E. \& Vavassori, P. Flexible spintronic devices on Kapton. Applied Physics Letters 104, 062412 (2014).

9. Barraud, C. et al. Magnetoresistance in magnetic tunnel junctions grown on flexible organic substrates. Applied Physics Letters 96, 072502 (2010).

10. Melzer, M. et al. Stretchable Magnetoelectronics. NanoLetters 11, 2522 (2011).

11. Melzer, M. et al. Wearable magnetic field sensors for flexible electronics. Advanced Materials 27, 1274 (2015).

12. Li, H. et al. Stretchable Spin Valve with Stable Magnetic Field Sensitivity by Ribbon-Patterned Periodic Wrinkles. ACS Nano 10, 4403 (2016).

13. Cordill, M. J., Glushko, O. \& Putz, B. Electro-Mechanical Testing of Conductive Materials Used in Flexible Electronics. Frontiers in Materials 2, 11 (2016)

14. Yu, Y. et al. Static and high frequency magnetic properties of FeGa thin films deposited on convex flexible substrates. Applied Physics Letters 106, 162405 (2015).

15. Gueye, M. et al. Effective 90-degree magnetization rotation in Co2FeAl thin film/piezoelectric system probed by microstripline ferromagnetic resonance. Applied Physics Letters 107, 032908 (2015).

16. Gueye, M. et al. Bending strain-tunable magnetic anisotropy in Co2FeAl Heusler thin film on Kapton ${ }^{\circledR}$. Appl. Phys. Letters $\mathbf{1 0 5}$, 062409 (2014).

17. Dundurs, J. W. Edge-bonded dissimilar orthogonal elastic wedges. J. App. Mech. 36, 650 (1969).

18. Schmauder, S. Theory of the Elastic Interface Crack. Ceramic Forum International 2, 101 (1987)

19. He, M. Y. \& Hutchinson, J. W. Crack deflection at an interface between dissimilar elastic materials. International Journal of Solids and Structures 25, 1053 (1989)

20. Guo, L., Ren, Y., Kong, L. Y., Chim, W. K. \& Chiam, S. Y. Ordered fragmentation of oxide thin films at submicron scale. Nature Communication 7, 13148 (2016).

21. Marthelot, J. et al. Self-replicating cracks: a collaborative fracture mode in thin films. Physical Review Letters 113, 085502 (2014).

22. Frank, S., Handge, U. A., Olliges, S. \& Spolenak, R. The relationship between thin film fragmentation and buckle formation: Synchrotron-based in situ studies and two-dimensional stress analysis. Acta Materialia 57, 1442 (2009).

23. Cordill, M. J., Fischer, F. D., Rammerstorfer, F. G. \& Dehm, G. Adhesion energies of Cr thin films on polyimide determined from buckling: Experiment and model. Acta Materialia 58, 5520 (2010).

24. Wu, K. et al. Buckling behaviors and adhesion energy of nanostructured $\mathrm{Cu} / \mathrm{X}(\mathrm{X}=\mathrm{Nb}, \mathrm{Zr})$ multilayer films on a compliant substrate. Acta Materialia 61, 7889 (2013).

25. Jörg, T. et al. Deformation behavior of Re alloyed Mo thin films on flexible substrates: In situ fragmentation analysis supported by first-principles calculations. Scientific Reports 7, 7374 (2017).

26. Tsymbal, E. Y. Spintronics: Electric toggling of magnets. Nature Materials 11, 12 (2012). 
27. Bonin, R., Schneidera, M. L., Silva, T. J. \& Nibarger, J. P. Dependence of magnetization dynamics on magnetostriction in NiFe alloys. Journal of Applied Physics 98, 123904 (2005).

28. Cordill, M. J., Glushko, O., Kreith, J., Marx, V. M. \& Kirchlechner, C. Measuring electro-mechanical properties of thin films on polymer substrates. Microelectron. Eng. 137, 96 (2015).

29. Faurie, D., Zighem, F., Garcia-Sanchez, A., Lupo, P. \& Adeyeye, A. O. Fragmentation and adhesion properties of CoFeB thin films on polyimide substrate. Applied Physics Letters 110(9), 091904 (2017).

30. Belmeguenai, M. et al. Annealing temperature and thickness dependencies of structural and magnetic properties of $\mathrm{Co} 2 \mathrm{FeAl}$ thin film. Physical Review B 94(10), 104424 (2016).

31. Zighem, F., Belmeguenai, M., Faurie, D., Haddadi, H. \& Moulin, J. Combining ferromagnetic resonator and digital image correlation to study the strain induced resonance tunability in magnetoelectric heterostructures. Review of Scientific Instruments 85, 103905 (2014).

32. Gueye, M. et al. Ferromagnetic resonance in thin films submitted to multiaxial stress state: application of the uniaxial equivalent stress concept and experimental validation. Journal of Physics D: Applied Physics 49(26), 265001 (2016).

33. Zighem, F. et al. Annealing effect on elastic, magnetic and magnetoelastic properties of $\mathrm{CoFeB}$ thin films on polymer substrate. Journal of Physics D: Applied Physics 50(45), 455002 (2016).

34. Frank, S., Gruber, P. A., Handge, U. A. \& Spolenak, R. In situ studies on the cohesive properties of a- and b-Ta layers on polyimide substrates. Acta Materialia 59, 5881 (2011).

35. Jansson, N. E., Leterrier, Y. \& Månson, J.-A. E. Modeling of multiple cracking and decohesion of a thin film on a polymer substrate. Engineering Fracture Mechanics 73, 2614 (2006).

36. Heinrich, B. \& Bland, J. A. C. Ultrathin magnetic structures II: Measurement techniques and novel magnetic properties, Eds, Springer-Verlag, Berlin, vol. 2 (1994).

37. Gueye, M., Lupo, P., Zighem, F., Faurie, D. \& Belmeguenai, M. Unambiguous magnetoelastic effect on residual anisotropy in thin films deposited on flexible substrates. A. O. Adeyeye, Europhysics Letters 114, 17003 (2017).

38. Zighem, F. et al. Micro-strip ferromagnetic resonance study of strain-induced anisotropy in amorphous FeCuNbSiB film on flexible substrate. Journal of Applied Physics 116(12), 123903 (2014).

39. Tang, Z. et al. Magneto-mechanical coupling effect in amorphous Co40Fe40B20 films grown on flexible substrates. Applied Physics Letters 105, 103504 (2014).

40. Pan, H. et al. Phononic and magnonic dispersions of surface waves on a permalloy/BARC nanostructured array. Nanoscale Research Letters 8, 115 (2013).

41. Gueye, M. et al. Spectroscopic investigation of elastic and magnetoelastic properties of CoFeB thin films. Journal of Physics D: Applied Physics 49, 145003 (2016).

42. Melzer, M., Lin, G., Makarov, D. \& Schmidt, O. G. Stretchable spin valves on elastomer membranes by predetermined periodic fracture and random wrinkling. Advanced Materials 24, 6468-6472 (2012).

43. Zighem, F. et al. Large area periodic ferromagnetic nanowires deposited onto a polymer substrate. Applied Physics Letters 111, 052408 (2017).

44. Suhl, H. Ferromagnetic Resonance in Nickel Ferrite Between One and Two Kilomegacycles. Physical Review 97, 555 (1955)

45. Goryunov, Y. V. et al. Magnetic anisotropies of sputtered Fe films on MgO substrates. Physical Review B 52, 13450 (1995).

46. Arias, R. \& Mills, D. L. Extrinsic contributions to the ferromagnetic resonance response of ultrathin films. Physical Review B 60, 7395 (1999).

\section{Acknowledgements}

Authors would like to thank Université Sorbonne Paris Cite and the National University of Singapore for their support through the USPC-NUS program (MagnoFlex project, ANR-11-IDEX-0005-02 and Nanoflex project, 2016-04-R/USPC-NUS).

\section{Author Contributions}

S.M., F.Z., A.G.S. and D.F. made in situ AFM exeriments. V.G., X.Z. and P.L. made thin film depositions. S.M., F.Z. and M.B. made F.M.R. experiments. Data interpretations were made by F.Z., A.O.A. and D.F. The manuscript was written by F.Z., A.O.A. and D.F. and edited by all authors before submission.

\section{Additional Information}

Competing Interests: The authors declare no competing interests.

Publisher's note: Springer Nature remains neutral with regard to jurisdictional claims in published maps and institutional affiliations.

Open Access This article is licensed under a Creative Commons Attribution 4.0 International License, which permits use, sharing, adaptation, distribution and reproduction in any medium or format, as long as you give appropriate credit to the original author(s) and the source, provide a link to the Creative Commons license, and indicate if changes were made. The images or other third party material in this article are included in the article's Creative Commons license, unless indicated otherwise in a credit line to the material. If material is not included in the article's Creative Commons license and your intended use is not permitted by statutory regulation or exceeds the permitted use, you will need to obtain permission directly from the copyright holder. To view a copy of this license, visit http://creativecommons.org/licenses/by/4.0/.

(C) The Author(s) 2018 